\title{
Universiteit
}

Leiden

The Netherlands

\section{Quantum-trajectory description of laser noise with depletion}

Visser, J.; Nienhuis, G.; Dutra, S.M.; Exter, M.P. van; Woerdman, J.P.

\section{Citation}

Visser, J., Nienhuis, G., Dutra, S. M., Exter, M. P. van, \& Woerdman, J. P. (2002). Quantumtrajectory description of laser noise with depletion. Physical Review A, 65, 063809. doi:10.1103/PhysRevA.65.063809

Version: $\quad$ Not Applicable (or Unknown)

License: $\quad$ Leiden University Non-exclusive license

Downloaded from: https://hdl.handle.net/1887/61356

Note: To cite this publication please use the final published version (if applicable). 


\title{
Quantum-trajectory description of laser noise with pump depletion
}

\author{
J. Visser, G. Nienhuis, S. M. Dutra, M. P. van Exter, and J. P. Woerdman \\ Huygens Laboratorium, Universiteit Leiden, Postbus 9504, 2300 RA Leiden, The Netherlands
}

(Received 13 September 2001; published 5 June 2002)

\begin{abstract}
The intensity fluctuations of a three-level laser are known to drop below shot noise in the presence of depletion of the ground state. We study the fluctuations of the laser output as a function of the parameter $\varepsilon$, defined as the fraction of atoms needed for the laser to operate. For a sufficiently small number of active atoms, the value of $\varepsilon$, and thereby the ground-state depletion, can be appreciable even for modest pumping. This suggests that the intensity fluctuations in the laser output would decrease below shot noise as the number of active atoms is reduced. A microscopic approach that uses a quantum-trajectory method and a macroscopic approach using semiclassical rate equations both show, however, that rather than decreasing, the intensity fluctuations actually increase with $\varepsilon$. We find that the fluctuations of the output are determined by the dependence of the cycle time of the atoms on the number of photons in the laser mode.
\end{abstract}

DOI: 10.1103/PhysRevA.65.063809

PACS number(s): 42.50.Lc, 42.50.Ar, 42.50.Dv

\section{INTRODUCTION}

One of the most important characteristics of laser radiation is its noise [1]. In recent years, it has been recognized that noise properties change when the pumping is strong enough to cause depletion of the number of atoms participating in the laser transition. The special case of a three-level laser with pump depletion has been studied [2,3]. Strong pumping leads to depletion of the ground state and the common assumption that the atoms in the ground state form an independent reservoir breaks down. It was found that for a laser with an incoherent pump, this depletion leads to an effective reduction of the pump noise, which in turn causes the intensity fluctuations of the laser output to drop below the shot-noise limit. However, the strong pumping needed for this mechanism to occur is far beyond experimental possibilities.

In this paper we consider a situation where depletion of the ground state is possible already with modest pumping. When the total number of atoms in the gain medium $N$ is not much larger than the inversion $N_{t h r}$ at threshold, groundstate depletion occurs already just above threshold, and extremely strong pumping is not required. This also raises the question whether the depletion of the ground state that occurs for atom numbers $N$ comparable to $N_{t h r}$ is also accompanied by intensity fluctuations below shot noise. If so, a Poissonian pumped laser with a sub-Poissonian output would be experimentally realizable.

As an example of a laser with a limited number of atoms, we analyze a three-level laser in a $\Lambda$ configuration, in the case where $N$ is allowed to be comparable to $N_{t h r}$. To find the intensity fluctuations we use a semiclassical rate equation for the number of photons in the laser mode with a noise source added. To account for the gain medium of the laser, a term $G_{a t}$ is added. An expression for $G_{a t}$ is obtained by using both a microscopic and a macroscopic approach to the dynamics of the gain medium. The optical Bloch equations are the starting point for both approaches.

The microscopic approach consists of a quantumtrajectory method to study the dynamics of a single atom in the gain medium. This allows us to derive the statistical properties of the stimulated emission of photons in the laser mode by a single atom. The dynamics of single atoms in the gain medium has been studied before by Ritsch and Zoller [4], but in their paper the atomic density matrix is calculated. A study of the intensity fluctuations in the fluorescence that accompanies quantum jumps in a driven three-level atom has been done by Kim and Knight [5]. From the dynamics of a single atom we derive the statistical properties of the photons deposited in the laser mode by the entire gain medium.

On the other hand, the macroscopic approach consists of a calculation based on semiclassical rate equations for the number of atoms in each level with noise sources added. For both approaches an expression for $G_{a t}$ is found and the intensity fluctuations of the laser output are calculated from the rate equation for the photon number. The fluctuations depend on the parameter $\varepsilon=N_{t h r} / N$, which represents the fraction of the number of atoms minimally needed for laser action. Both the microscopic and the macroscopic approaches bring us to the conclusion that large values of $\varepsilon$ do not lead to intensity fluctuations below shot noise.

In the following section we discuss the rate equation for the photon number, and the optical Bloch equations that describe the interaction between the laser mode and the gain medium. In Sec. III we discuss the microscopic approach that uses quantum trajectories to describe the evolution of a single atom in the gain medium. The macroscopic approach, for which semiclassical rate equations for the number of atoms in each level are derived, is discussed in Sec. IV. Conclusions are given in Sec. V.

\section{OPTICAL BLOCH EQUATIONS}

\section{A. Laser model}

We model a laser by a laser mode in a cavity that is resonant with the lasing transition of the atoms in the gain medium. We use three-level atoms in the $\Lambda$ configuration, as depicted in Fig. 1. The lasing transition 2-1 is coherently driven by the laser mode. Spontaneous emission occurs from state 2 to state 1 , and from state 1 to state 0 , and the corresponding rates are $\gamma_{2}$ and $\gamma_{1}$. The lower state 0 is incoher- 


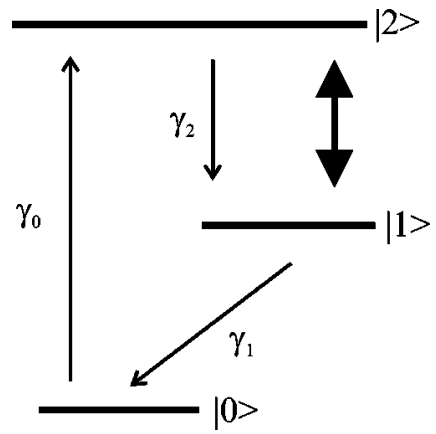

FIG. 1. The three-level scheme, where the 2-1 transition is the lasing transition.

ently pumped to the upper level 2 at the rate $\gamma_{0}$. For the number of photons in the laser mode we use the following rate equation:

$$
\dot{n}=-2 \kappa n+G_{a t}(n)+f_{n},
$$

where $\kappa$ is the cavity decay rate. The function $G_{a t}(n)$ models the effect the atoms in the gain medium have on the number of photons in the laser mode. It can be obtained from a detailed description of the interaction of the atoms in the gain medium with the photons in the laser mode. Because $n$ is not a continuous but an integer number, the creation and annihilation of photons in the cavity introduces noise, which is accounted for by the noise source $f_{n}$.

The evolution of the density matrix of a three-level atom coherently coupled to the laser mode is described by the optical Bloch equations. We have

$$
\begin{gathered}
\frac{d}{d t} \rho_{22}=-\gamma_{2} \rho_{22}+\gamma_{0} \rho_{00}+\frac{i}{2} \Omega\left(\rho_{12}-\rho_{21}\right), \\
\frac{d}{d t} \rho_{11}=-\gamma_{1} \rho_{11}+\gamma_{2} \rho_{22}+\frac{i}{2} \Omega\left(\rho_{21}-\rho_{12}\right), \\
\frac{d}{d t} \rho_{21}=-\gamma_{\perp} \rho_{21}+\frac{i}{2} \Omega\left(\rho_{11}-\rho_{22}\right)=\frac{d}{d t} \rho_{12}^{*}, \\
\frac{d}{d t} \rho_{00}=-\frac{d}{d t} \rho_{22}-\frac{d}{d t} \rho_{11} .
\end{gathered}
$$

We neglect collisional dephasing, so that the optical coherence on the lasing transition decays at the transverse rate

$$
\gamma_{\perp}=\frac{1}{2}\left(\gamma_{1}+\gamma_{2}\right)
$$

No optical coherence involving the state 0 is created. This scheme can be expected to give rise to laser action only when the lower state of the lasing transition decays much faster than the upper state, so that $\gamma_{1} \gg \gamma_{2}$. The coupling of the lasing transition to the laser mode is modeled by an effective Rabi frequency $\Omega$. Adiabatic elimination of the optical coherence $\rho_{21}$ shows that the effective rate of stimulated emission is

$$
\gamma_{s t}=\frac{\Omega^{2}}{2 \gamma_{\perp}} .
$$

This rate is related to the average photon number $\bar{n}$ in the laser mode by the equality $\gamma_{s t}=\gamma_{2} \beta \bar{n}$, with $\beta$ being the fraction of spontaneously emitted photons on the laser transition that go into the laser mode. This connects the Rabi frequency to the properties of the laser cavity.

\section{B. The fraction of atoms needed for lasing}

In a laser the stimulated emission rate by the whole gain medium is equal to $\gamma_{2} \beta n N_{2}$, where $N_{2}$ is the number of atoms in level 2. The absorption rate is $\gamma_{2} \beta n N_{1}$, where $N_{1}$ is the number of atoms in level 1 . The decay rate of photons from the cavity is $2 \kappa n$. In the steady state above threshold we find

$$
\gamma_{2} \beta \bar{n}\left(\bar{N}_{2}-\bar{N}_{1}\right)=2 \kappa \bar{n}
$$

where the quantities with a bar refer to the steady-state values. For the inversion we find

$$
\bar{D}=\bar{N}_{2}-\bar{N}_{1}=\frac{2 \kappa}{\gamma_{2} \beta}=N_{t h r} .
$$

The number of atoms that is at least needed for the laser to operate is then equal to $N_{t h r}$. We define $\varepsilon$ as the fraction of the total number of atoms that is at least needed for the laser to operate, that is

$$
\varepsilon=\frac{N_{t h r}}{N}
$$

where $N$ is the total number of atoms in the gain medium.

In the laser we distinguish a limited number of uncorrelated sources of noise in the photon number. We use $f_{s t}$ for stimulated emission, $f_{a b s}$ for absorption, and $f_{v a c}$ for the fluctuations related to the emission of photons from the cavity. The relation between the noise term $f_{n}$ in Eq. (1) and the uncorrelated noise terms is given by

$$
f_{n}=f_{s t}-f_{a b s}-f_{v a c},
$$

where the signs reflect the gain and loss nature of the noise process for the variable involved. We assume that the noise sources are Gaussian and $\delta$-correlated random variables with zero average. For the correlations between the noise sources we have

$$
\left\langle f_{i}(t) f_{j}\left(t^{\prime}\right)\right\rangle=D_{i j} \delta\left(t-t^{\prime}\right),
$$

where $D_{i j}$ are the diffusion coefficients. Since the noise sources are uncorrelated, the only nonzero diffusion coefficients are the diagonal ones, which we indicate as $D_{s t}$, $D_{a b s}$, and $D_{v a c}$. According to Lax [6] these diffusion terms are equal to the corresponding rate, so that

$$
D_{s t}=\gamma_{2} \beta \bar{n} \bar{N}_{2} \text {, }
$$




$$
\begin{gathered}
D_{a b s}=\gamma_{2} \beta \bar{n} \bar{N}_{1}, \\
D_{v a c}=2 \kappa \bar{n} .
\end{gathered}
$$

\section{Spectrum of intensity fluctuations}

We are interested in the spectrum $V(\omega)$ of the intensity fluctuations at the frequency $\omega=0$. The spectrum $V(\omega)$ is defined as follows:

$$
\left\langle\overline{\Delta I}(\omega) \overline{\Delta I}\left(\omega^{\prime}\right) *\right\rangle=2 \kappa \bar{n} V(\omega) \delta\left(\omega-\omega^{\prime}\right)
$$

where

$$
\overline{\Delta I}(\omega)=\frac{1}{\sqrt{2 \pi}} \int_{-\infty}^{\infty} d t \exp (-i \omega t) \Delta I(t)
$$

is the Fourier transform of the fluctuations $\Delta I(t)$ of the output intensity of the laser around the steady-state value $\bar{I}$ $=2 \kappa \bar{n}$. To find $\Delta I(t)$ we linearize Eq. (1) around the steady state by substituting $n(t)=\bar{n}+\Delta n(t)$ and neglecting terms that are second order in $\Delta n$ or higher. The next step is to relate the internal fluctuations in the photon number to the fluctuations in the output intensity. Therefore we use the input-output formalism of Gardiner and Collett [7]. In their paper they use the standard model of a system coupled to a heat bath. They derive a boundary condition that relates the input and the output to the internal modes of the system. In our case the input to the system is the vacuum state and the output is the intensity of the laser. We find

$$
\Delta I(t)=2 \kappa \Delta n(t)+f_{v a c}(t) .
$$

We study the dependence of the intensity fluctuations on the parameter $\varepsilon$ by using, respectively, a microscopic and a macroscopic approach to describe the effect of the gain medium on the number of photons in the laser mode. We determine the function $G_{a t}(n)$ that accounts for the gain medium in the rate equation for $n \mathrm{Eq}$. (1) and use the approach given above to find an expression for the spectrum of the intensity fluctuations at frequency $\omega=0$ as a function of $\varepsilon$. In both cases the optical Bloch equations (2) are the starting point.

\section{QUANTUM TRAJECTORIES}

\section{A. Model description}

In order to determine the function $G_{a t}(n)$ that accounts for the photon gain in the laser mode, we analyze in the present section the statistical properties of the cycles performed by the internal state of the atoms of the gain medium. This is done by the technique of quantum trajectories, in which the density matrix of each atom is unraveled into an ensemble of pure states [8-10]. A trajectory description of lasers without inversion has been given before [11]. Spontaneous emission and pumping constitute the incoherent transitions in this model, which can be described by quantum jumps. The corresponding jump operators are $S_{2}=|1\rangle\langle 2|$,
$S_{1}=|0\rangle\langle 1|$, and $S_{0}=|2\rangle\langle 0|$. The optical Bloch equations (2) correspond to the master equation

$$
\frac{d}{d t} \rho=-i\left(H \rho-\rho H^{\dagger}\right)+\gamma_{2} S_{2} \rho S_{2}^{\dagger}+\gamma_{1} S_{1} \rho S_{1}^{\dagger}+\gamma_{0} S_{0} \rho S_{0}^{\dagger},
$$

with $H$ the effective non-Hermitian Hamiltonian

$$
H=-\frac{1}{2}\left[\left(\Omega\left(S_{2}+S_{2}^{\dagger}\right)+i \gamma_{0}|0\rangle\left\langle 0\left|+i \gamma_{1}\right| 1\right\rangle\left\langle 1\left|+i \gamma_{2}\right| 2\right\rangle\langle 2|\right] .\right.
$$

Atoms are performing cycles $0 \rightarrow 2 \rightarrow 1 \rightarrow 0$ along their internal states, thereby depositing photons into the laser mode when stimulated emission occurs. In order to describe the atomic contribution to the noise in the photon number, we consider the possible histories of an atom during a cycle. The atom undergoes coherent evolution periods, described by the effective Hamiltonian $H$. The coherent evolution is interrupted by instantaneous quantum jumps, described by the jump operators $S_{i}$ with $i=0,1,2$. Solutions of the master equation (11), or, equivalently, the optical Bloch equations (2) are faithfully reproduced after averaging these single histories over the instants of the jumps $[8,9]$ with the proper probability distribution. We follow the atom during one cycle $0 \rightarrow 2 \rightarrow 1 \rightarrow 0$, which is the time period between two successive arrivals of the atom in state 0 by spontaneous emission from the state 1 . After a lifetime of average duration $\gamma_{0}^{-1}$ in 0 , the atom is pumped to the upper state 2 , as described by $S_{0}$, and the cycle ends with spontaneous decay from 1 to 0 , expressed by $S_{1}$. In between these two jump instants, the atom undergoes coherent evolution within the two-state manifold 1 and 2, and any number $k$ of spontaneous decays from 2 to 1 , as described by the jump operator $S_{2}$.

\section{B. Statistics of cycle trajectories}

We denote a period of coherent evolution starting in state $i$ and ending in state $j$ as $(i, j)$. An atom starting a coherent period in state 0 can only remain in this state, and the only possible coherent period is $(0,0)$. A coherent period starting in state 2 or in state 1 can end either in 2 or 1 , with the corresponding periods $(2,2),(2,1),(1,2)$, or $(1,1)$. Therefore, the probabilities $P_{i j}$ that a coherent period starting in state $i$ ends in state $j$ obey the obvious sum rules

$$
P_{00}=1, P_{11}+P_{12}=1, P_{21}+P_{22}=1 \text {. }
$$

The average duration time of a coherent period $(i, j)$ is indicated as $T_{i j}$.

In Fig. 2 the possible trajectories for cycles $0 \rightarrow 2 \rightarrow 1$ $\rightarrow 0$ are depicted, where the number $k$ indicates the number of spontaneous emissions during the cycle. Each spontaneous emission has a small probability $\beta$ to return a photon to the laser mode. Stimulated emission into the laser mode takes place only during the coherent period $(2,1)$. Such a period occurs only in the first trajectory in Fig. 2 with $k$ $=0$. This trajectory is termed the gain trajectory, and we shall demonstrate that it becomes dominant above threshold. For nonzero values of the number $k$ of spontaneous decays on the laser transition, the trajectory contains $k-1$ absorp- 


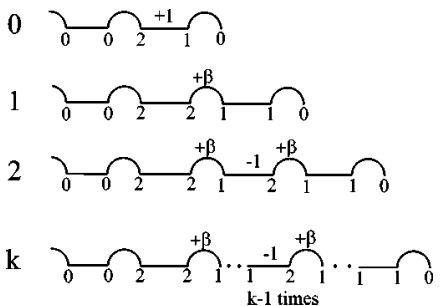

FIG. 2. The different trajectories an atom can follow during one cycle, starting in the ground-state level 0 . The trajectories are labeled on the left. An arc represents a quantum jump and a straight line a coherent period. The state of the atom is given at the beginning and the end of each coherent period. The net effect of a coherent period or a quantum jump on the photon number is also given.

tions of a photon from the laser mode, each one corresponding to a coherent period $(1,2)$. The passage through the manifold of lasing states starts with a coherent period $(2,2)$, and ends with a coherent period $(1,1)$.

The probability distribution $p_{k}$ over trajectories with $k$ spontaneous emissions on the lasing transition can be read off from Fig. 2, with the result

$$
p_{0}=P_{21}, \quad p_{k}=P_{22} P_{12}^{k-1} P_{11} \text { for } k \geqslant 1 \text {. }
$$

By using the sum rules (12) it is easy to check that the probabilities add up to 1 . Using these probabilities the average value of interesting physical quantities, such as the average duration of a cycle, can be calculated. The average duration $T_{k}$ of a trajectory with $k$ spontaneous emissions is simply the sum over the average durations of the coherent periods, so that

$T_{0}=T_{00}+T_{21}, \quad T_{k}=T_{00}+T_{22}+(k-1) T_{12}+T_{11}$ for $k \geqslant 1$.

By using the sum rules (12), and the expressions (13) and (14) for $p_{k}$ and $T_{k}$, the average duration of a cycle $T_{\text {cycle }}$ $=\Sigma_{k} p_{k} T_{k}$ can be simplified to the form

$$
T_{\text {cycle }}=T_{00}+P_{21} T_{21}+P_{22}\left(T_{11}+T_{22}\right)+\frac{P_{22}}{P_{11}} P_{12} T_{12} .
$$

In order to evaluate the probabilities $P_{i j}$ and the average durations $T_{i j}$, we notice that for a given initial density matrix $\rho_{0}$, the time-dependent density matrix $\tilde{\rho}(\tau)=\exp$ $(-i H \tau) \rho_{0} \exp \left(i H^{\dagger} \tau\right)$ constitutes the partial density matrix for the situation that no quantum jump occurred during the time interval $[0, \tau]$. Its trace $\operatorname{Tr} \tilde{\rho}$ represents the corresponding probability that no quantum jump occurred in $[0, \tau]$. We introduce the decaying transition amplitudes

$$
c_{i j}(\tau)=\langle j|\exp (-i H \tau)| i\rangle,
$$

such that the quantity $\gamma_{j}\left|c_{i j}(\tau)\right|^{2}$ is the probability density that a coherent period that started in the state $i$ ends after a time duration $\tau$ with a jump from the state $j$. This leads to the expression

$$
P_{i j}=\gamma_{j} \int_{0}^{\infty} d \tau\left|c_{i j}(\tau)\right|^{2}
$$

for the probability of a coherent period $(i, j)$ under the condition that it started in state $i$. We define $L_{i j}(\tau)$ as the normalized probability density that a coherent period $(i, j)$ ends after a time duration $\tau$ and find

$$
L_{i j}(\tau)=\gamma_{j}\left|c_{i j}(\tau)\right|^{2} / P_{i j}
$$

For the average duration time of a coherent period $(i, j)$ we find

$$
T_{i j}=\int_{0}^{\infty} d \tau L_{i j}(\tau) \tau
$$

Obviously, for the coherent period $(0,0)$ in the lower state we recover that $P_{00}=1$, and $T_{00}=\gamma_{0}^{-1}$. For the transition amplitudes within the manifold of the laser states, we obtain the results

$$
\begin{aligned}
c_{22}(\tau)= & \frac{1}{2} \exp \left(-\frac{1}{4}\left(\gamma_{1}+\gamma_{2}\right) \tau\right)\left[\left(1+\frac{\gamma_{1}-\gamma_{2}}{4 \Gamma}\right) \exp (\Gamma \tau)\right. \\
& \left.+\left(1-\frac{\gamma_{1}-\gamma_{2}}{4 \Gamma}\right) \exp (-\Gamma \tau)\right], \\
c_{11}(\tau)= & \frac{1}{2} \exp \left(-\frac{1}{4}\left(\gamma_{1}+\gamma_{2}\right) \tau\right)\left[\left(1-\frac{\gamma_{1}-\gamma_{2}}{4 \Gamma}\right) \exp (\Gamma \tau)\right. \\
& \left.+\left(1+\frac{\gamma_{1}-\gamma_{2}}{4 \Gamma}\right) \exp (-\Gamma \tau)\right], \\
c_{21}(\tau)= & c_{12}(\tau)=i \frac{\Omega}{4 \Gamma} \exp \left(-\frac{1}{4}\left(\gamma_{1}+\gamma_{2}\right) \tau\right) \\
& \times[\exp (\Gamma \tau)-\exp (-\Gamma \tau)],
\end{aligned}
$$

where

$$
\Gamma=\frac{1}{4} \sqrt{\left(\gamma_{1}-\gamma_{2}\right)^{2}-4 \Omega^{2}} .
$$

These expressions (17) are generally valid for the coherent evolution of the states coupled by the lasing transition. The Rabi frequency $\Omega$ is related to the photon number by

$$
\frac{\Omega^{2}}{2 \gamma_{\perp}}=\gamma_{2} \beta n,
$$

as argued below Eq. (4).

\section{Cycle time of the gain trajectory}

As argued above, the only trajectory leading to stimulated emission is the first one in Fig. 2 (with $k=0$ ). Using the results of the previous section, we calculate the probability $p_{0}$ of this first trajectory and find

$$
p_{0}=P_{21}=\frac{\gamma_{1} \gamma_{2} \beta n}{\gamma_{1} \gamma_{2}+\left(\gamma_{1}+\gamma_{2}\right) \gamma_{2} \beta n} \text {. }
$$


Far above the threshold it is known that $\beta n \gg 1$, and since $\gamma_{1} \gg \gamma_{2}$ we see that the probability of the gain trajectory $p_{0}$ is close to 1 . Therefore we assume that far above the threshold, atoms in the gain medium of the laser follow only the gain trajectory.

During a coherent period the photon number is not known, because of the entanglement with the states of the atom. Therefore we cannot describe the gain of the photon in the gain trajectory as an event that takes place at a specific time $t$. For simplicity we assume that the actual gain of the photon takes place at the end of the coherent period $(2,1)$. Far above the threshold, the atom follows the gain trajectory and we can view the atom as a source that emits photons in the laser mode with varying time intervals between two consecutive emissions. We define $w_{1}(\tau)$ as the normalized waiting-time distribution for the creation of the next photon. Since, between the emissions of two consecutive photons the atom evolves through the coherent periods $(0,0)$ and $(2,1)$, we have

$$
w_{1}(\tau)=\int_{0}^{\tau} d t L_{21}(\tau-t) L_{00}(t),
$$

where the $L_{i j}(\tau)$ are the normalized probability densities defined in Eq. (15). Because directly after the emission of a photon it takes time for the atom to get excited again, the emission of a single atom in the gain medium is antibunched as can be seen from Eq. (18).

We define $f_{1}\left(\tau_{1}\right) d \tau_{1}$ as the probability that there is an emission of a photon in the time interval $\left(\tau_{1}, \tau_{1}+d \tau_{1}\right)$, regardless of how many photons have been emitted outside this time interval [12]. The expectation value of the number $m$ of emitted photons in the time interval $(0, T)$ is given by

$$
\langle m\rangle_{T}=\int_{0}^{T} d \tau_{1} f_{1}\left(\tau_{1}\right)
$$

If we divide $\langle m\rangle_{T}$ by the time $T$, we have the average intensity. In the limit of $T \rightarrow \infty$ we have

$$
\lim _{T \rightarrow \infty} \frac{\langle m\rangle_{T}}{T}=f_{1}(\infty),
$$

which is the steady-state emission rate. The Laplace transform $\hat{f}$ of a function $f$ is defined as

$$
\hat{f}(s)=\int_{0}^{\infty} d \tau \exp (-s \tau) f(\tau) .
$$

Using Eqs. (21) and (20) we derive that

$$
f_{1}(\infty)=\lim _{s \rightarrow 0} s \hat{f}_{1}(s) .
$$

Since a photon emitted at time $\tau_{1}$ can be the first, the second, and so on after time 0 , the relation between the emission rate $f_{1}\left(\tau_{1}\right)$ and the waiting-time distribution $w_{1}\left(\tau_{1}\right)$ in Laplacetransform language takes the form [13]

$$
\hat{f}_{1}(s)=\hat{w}_{1}(s)+\left[\hat{w}_{1}(s)\right]^{2}+\cdots=\frac{\hat{w}_{1}(s)}{1-\hat{w}_{1}(s)} .
$$

From Eq. (18) we find that

$$
\hat{w}_{1}(s)=\hat{L}_{21}(s) \hat{L}_{00}(s) .
$$

Using Eq. (22) we find

$$
\frac{1}{f_{1}(\infty)}=-\lim _{s \rightarrow 0} \frac{d}{d s} \hat{w}_{1}(s)=\int_{0}^{\infty} d \tau w_{1}(\tau) \tau,
$$

which is equal to the average time between the emissions of two consecutive photons as expected. Since the atoms follow only the gain trajectory, we may write $f_{1}(\infty)=1 / T_{0}$. Using Eq. (23) we find

$$
\begin{aligned}
\frac{1}{f_{1}(\infty)} & =T_{0}=-\lim _{s \rightarrow 0} \frac{d}{d s} \hat{w}_{1}(s)=-\lim _{s \rightarrow 0} \frac{d}{d s}\left[\hat{L}_{21}(s) \hat{L}_{00}(s)\right] \\
& =T_{00}+T_{21},
\end{aligned}
$$

as expected from Fig. 2. Using Eq. (24) together with the expression (16) for $T_{i j}$ we find for $f_{1}(\infty)$ the following expression:

$$
\frac{1}{f_{1}(\infty)}=\frac{1}{\gamma_{0}}+\frac{2}{\gamma_{1}+\gamma_{2}}+\frac{\gamma_{1}+\gamma_{2}}{\gamma_{1} \gamma_{2}+\left(\gamma_{1}+\gamma_{2}\right) \gamma_{2} \beta n} .
$$

\section{Dynamics of a laser}

In the steady state the number of photons created per second by the gain medium of $N$ atoms is equal to the number of photons annihilated per second by cavity decay. Far above the threshold, we have

$$
N f_{1}(\infty)=2 \kappa \bar{n},
$$

where $\bar{n}$ is the steady-state number of photons. We make the following assumptions:

$$
\begin{gathered}
\beta n \gg 1, \\
\gamma_{2} \beta n \ll \gamma_{1},
\end{gathered}
$$

which define a range of pump rates far above the threshold for which the number of photons in the laser mode is proportional to the pump rate $\gamma_{0}$. Under these assumptions we have

$$
\frac{1}{f_{1}(\infty)}=T_{0}=\frac{1}{\gamma_{0}}+\frac{1}{\gamma_{2} \beta n} .
$$

We solve for $\bar{n}$ in Eq. (26) and find

$$
\bar{n}=\frac{\gamma_{0}}{\gamma_{2} \beta} \frac{1-\varepsilon}{\varepsilon},
$$

where $\varepsilon$, defined in Eq. (5), is the fraction of atoms that is at least needed for the laser to operate. From Eq. (29) we find that in general the assumptions (27) are satisfied for the range of pump rates for which we have $\gamma_{2} \ll \gamma_{0} / \varepsilon \ll \gamma_{1}$. 
We calculate the average cycle time of an atom far above the threshold, which is equal to the average duration time of the gain trajectory $T_{0}$ given in Eq. (24). Under the present assumptions and using the steady-state value (29) for $n$, we find

$$
T_{0}=\frac{1}{\gamma_{0}(1-\varepsilon)} .
$$

We see that the cycle time increases when $\varepsilon$ is increased. This is because for a fixed pump rate the number of photons in the laser mode decreases if $\varepsilon$ increases. Then the Rabi frequency becomes smaller and it takes more time for the atoms to make the 2-1 transition. Each cycle results in the gain of one photon in the laser mode. Thus if $\varepsilon$ increases and thereby the cycle time, the atoms become less productive in creating photons.

\section{E. Intensity fluctuations}

The number of atoms in the gain medium is a macroscopically large number. If we assume that there is no correlation between the atoms, the creation of photons in the laser mode by the gain medium as a whole, is a random process. The emission rate at time $t$ is then given by $N f_{1}(\infty)$, which is time dependent because it is a function of $n(t)$ as we see in Eq. (28). So in the rate equation (1) for $n$ we substitute $G_{a t}(n)=N f_{1}(\infty)$ and we obtain

$$
\dot{n}=-2 \kappa n+N\left(\frac{1}{\gamma_{0}}+\frac{1}{\gamma_{2} \beta n}\right)^{-1}+f_{n} .
$$

As discussed in Sec. II we linearize around the steady state. From Eq. (28) we find

$$
f_{1}(\infty)=\left(\frac{1}{\gamma_{0}}+\frac{1}{\gamma_{2} \beta \bar{n}}\right)^{-1}+\frac{2 \kappa \varepsilon}{N} \Delta n+\mathcal{O}\left(\Delta n^{2}\right),
$$

and Eq. (31) gives for the fluctuations $\Delta n(t)$ in the photon number

$$
\Delta \dot{n}=-2 \kappa(1-\varepsilon) \Delta n+f_{n},
$$

with the solution

$$
\Delta n(t)=\int_{-\infty}^{t} d t^{\prime} \exp \left[-2 \kappa(1-\varepsilon)\left(t-t^{\prime}\right)\right] f_{n}\left(t^{\prime}\right) .
$$

According to Eq. (10) we have $\Delta I(t)=2 \kappa \Delta n(t)+f_{\text {vac }}(t)$. The noise source $f_{n}$ is related to the uncorrelated noise sources in Eq. (6). The corresponding diffusion coefficients are given in Eq. (8). Because of the assumptions (27), the decay rate $\gamma_{1}$ of the lower lasing level is much larger than the other rates in the model. For the evaluation of the diffusion coefficients it is, therefore, justified to neglect the number of atoms in level 1 , and so that $\bar{N}_{1}=0$ and $\bar{N}_{2}=\bar{D}$ $=N_{t h r}$. We use the Whiener-Khintchine theorem [14], that is,

$$
\begin{aligned}
\left\langle\widetilde{\Delta I}(\omega) \widetilde{\Delta I}\left(\omega^{\prime}\right)^{*}\right\rangle= & \delta\left(\omega-\omega^{\prime}\right) \lim _{T \rightarrow \infty} \int_{-\infty}^{\infty} d \tau \exp (-i \omega \tau) \\
& \times\langle\Delta I(T+\tau) \Delta I(T)\rangle,
\end{aligned}
$$

and obtain the following expression for the spectrum of intensity fluctuations as defined in Eq. (9):

$$
V(\omega)=1+\frac{8 \kappa^{2} \varepsilon}{4 \kappa^{2}(1-\varepsilon)^{2}+\omega^{2}} .
$$

For the spectrum at frequency $\omega=0$ we have

$$
V(0)=1+\frac{2 \varepsilon}{(1-\varepsilon)^{2}} .
$$

The value $V(0)=1$ corresponds to shot noise in our definition (9). We see that for $\varepsilon=0$, which is the case of no depletion of the ground state at all, we have shot noise. If $\varepsilon$ is increased, the fluctuations rise above shot noise.

We can see this also from a qualitative argument. The average cycle time of the atom is $T_{0}=1 / f_{1}(\infty)$. If we calculate the average fraction of the total cycle time that the atom spends in the ground state we find, using Eq. (30),

$$
\frac{T_{00}}{T_{0}}=\frac{1}{\gamma_{0} T_{0}}=1-\varepsilon .
$$

We see that for small $\varepsilon$ the atom is in the ground state for most of the time. In that case the cycle time is not sensitive to fluctuations in the photon number. If $\varepsilon$ is increased, the atom spends less time in the ground state and a fluctuation in the photon number will cause more fluctuations in the cycle time.

In the Appendix the Mandel $Q$ parameter [15] is calculated for the emission of a single atom in the gain medium that follows only the gain trajectory. It is defined by $Q+1$ $=\Delta m^{2} /\langle m\rangle$, where $m$ is the number of photon emissions. We have $Q=0$ for a Poisson process. Under the assumptions (27) and using Eq. (29) it is found that

$$
Q=-2 \varepsilon(1-\varepsilon) .
$$

We see that for $\varepsilon=0$ we have $Q=0$ and the emission of a single atom is Poissonian. This is expected from the fact that for $\varepsilon=0$ the atom spends (almost) all its time in the ground state and thus the statistics of its emission are determined by the statistics of the pump, which are Poissonian. For $\varepsilon=0$ the output fluctuations are at shot-noise level. If $\varepsilon$ is increased, the value of $Q$ decreases until a minimum value of $-\frac{1}{2}$ is reached for $\varepsilon=\frac{1}{2}$. We can understand this from the fact that for $\varepsilon=\frac{1}{2}$ we have $T_{00}=T_{21}$, so that the cycle contains two coherent periods with the same average duration. According to Ritsch and Zoller [4] this regular recycling of the atom is accompanied by antibunching and subPoissonian emission. It is surprising that for nonzero $\varepsilon$ the emission of a single atom is sub-Poissonian, while the output fluctuations are above shot noise. 


\section{SEMICLASSICAL RATE EQUATIONS}

\section{A. Steady state}

In this section we study the steady-state and noise properties of the three-level laser from a macroscopic point of view using semiclassical rate equations. We assume that the total number of active atoms in the gain medium is constant and given by $N$, a macroscopically large number. Classical Langevin rate equations for the number of atoms in the different levels $N_{2}, N_{1}, N_{0}$ follow from the optical Bloch equations (2) where we adiabatically eliminate the atomic coherences of the lasing transition. We identify $N \rho_{i i}$ as the number of atoms $N_{i}$ in level $i$, which is justified for macroscopic $N$, and add Langevin noise terms. We find

$$
\begin{gathered}
\dot{N}_{2}=-\gamma_{2} N_{2}-\gamma_{2} \beta n\left(N_{2}-N_{1}\right)+\gamma_{0} N_{0}+f_{2}, \\
\dot{N}_{1}=-\gamma_{1} N_{1}+\gamma_{2} \beta n\left(N_{2}-N_{1}\right)+\gamma_{2} N_{2}+f_{1}, \\
\dot{N}_{0}=-\gamma_{0} N_{0}+\gamma_{1} N_{1}+f_{0},
\end{gathered}
$$

with $n$ the number of photons in the laser mode and $\beta$ the fraction of spontaneously emitted photons on the laser transition that go into the laser mode, as mentioned in Sec. II. The fraction $\beta$ is an independent parameter in this configuration. The terms $\gamma_{2} N_{2}$ and $\gamma_{1} N_{1}$ describe spontaneous emission from levels 2 and 1 , respectively, and the terms $\gamma_{2} \beta n N_{2}$ and $\gamma_{2} \beta n N_{1}$ represent stimulated emission and absorption. From this we derive the rate equation for the number of photons in the laser mode. We assume operation in the good-cavity regime, by which we mean that the cavity decay rate $2 \kappa$ is much smaller than the transverse relaxation rate $\gamma_{\perp}$ as given in Eq. (3). Under this assumption we find

$$
\dot{n}=-2 \kappa n+\gamma_{2} \beta\left[N_{2}(n+1)-N_{1} n\right]+f_{n} .
$$

If we compare this expression with Eq. (1) we can identify $G_{a t}(n)$ as the second term on the right. The noise sources $f_{i}$ with $i=n, 0,1,2$ are Gaussian $\delta$-correlated random variables with zero average. They appear in the rate equations because the quantities that are involved are not continuous numbers, but refer to an integer number such as the number of atoms for example. In Eq. (34) the factor $n+1$ instead of $n$ accounts for spontaneous emission in the laser mode. The rate equations (33) and (34) can also be derived directly from phenomenological considerations of the dynamics of a laser. The constraint on the total number of atoms is given by

$$
N_{0}(t)+N_{1}(t)+N_{2}(t)=N \text {. }
$$

The steady-state photon number $\bar{n}$ and inversion $\bar{D}=\bar{N}_{2}$ $-\bar{N}_{1}$ can be found from Eqs. (33) and (34) by setting the time derivatives to zero and omitting the noise sources. If we assume that $\beta \ll 1$ we find

$$
\bar{n}=\frac{1}{2 \beta}\left(M-1+\sqrt{(M-1)^{2}+4 \frac{\beta M}{1-\varepsilon} \frac{\gamma_{1}}{\gamma_{1}-\gamma_{2}}}\right),
$$

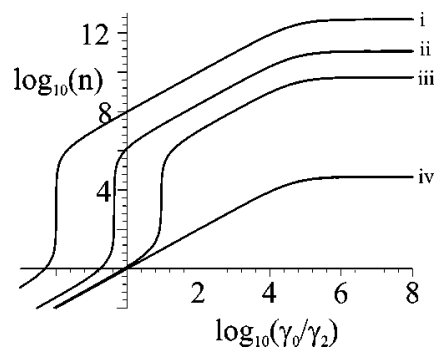

FIG. 3. Steady-state photon number $n$ as a function of the pump rate $\gamma_{0}$ in units of $\gamma_{2}$. We have (i) $\varepsilon=0.01$, (ii) $\varepsilon=0.3$, (iii) $\varepsilon$ $=0.9$, (iv) $\varepsilon=1$.

$$
\begin{aligned}
& M=\frac{1-\varepsilon}{\varepsilon} \frac{\gamma_{0}}{\gamma_{2}} \frac{\gamma_{1}-\gamma_{2}}{\gamma_{1}+2 \gamma_{0}}, \\
& \bar{D}=N \varepsilon \frac{\bar{n}\left(\gamma_{1}-\gamma_{2}\right)}{\bar{n}\left(\gamma_{1}-\gamma_{2}\right)+\gamma_{1}},
\end{aligned}
$$

where the parameter $\varepsilon$ defined in Eq. (5) again has the significance of the fraction of the total number of atoms that is at least needed for the laser to operate. Above threshold, where $\bar{n} \gg 1$, the inversion $\bar{D}$ equals $N_{t h r}$. The parameter $M$ is the pump parameter that is normalized to 1 at the threshold. In all the figures we use the values $\beta=10^{-6}$ and $\gamma_{2} / \gamma_{1}=10^{-5}$, which are characteristic for a typical $\mathrm{Nd}: \mathrm{YVO}_{4}$ laser.

In Fig. 3 the steady-state photon number $\bar{n}$ as a function of the pump rate is given for different values of $\varepsilon$. We see that when $\varepsilon$ is increased, the threshold moves to a higher pump rate. This is because just above the threshold the population in the ground state is $1-\varepsilon$. An increase in $\varepsilon$ is thus an increase in the depletion of the ground state, which must be compensated by a higher pump rate. The photon number stops increasing when the pump rate becomes of the same order of magnitude as the decay rate $\gamma_{1}$ of level 1 . Then the 1-0 transition starts to act as a bottleneck, with the consequence that a further increase in the pump rate increases the population in level 1 and depletes the ground state.

If we multiply the steady-state photon number $\bar{n}$ with the cavity decay rate $2 \kappa$ and divide by the total number of atoms $N$, we have the steady-state output intensity per atom. In Fig. 4 this quantity is given as a function of the pump rate for different values of $\varepsilon$. We see that the atoms become less productive when $\varepsilon$ is increased. This is understood from the

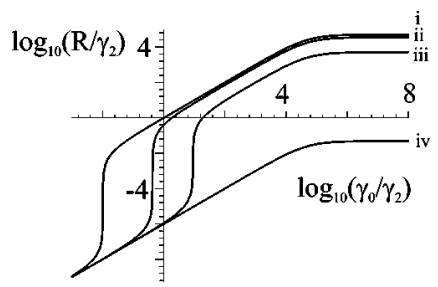

FIG. 4. Steady-state output intensity per atom $R=2 \kappa \bar{n} / N$ in units of $\gamma_{2}$ as a function of the pump rate $\gamma_{0}$ in units of $\gamma_{2}$. We have (i) $\varepsilon=0.01$, (ii) $\varepsilon=0.3$, (iii) $\varepsilon=0.9$, (iv) $\varepsilon=1$. 
discussion below Eq. (30), where it was found that the cycle time of an atom increases if $\varepsilon$ is increased.

\section{B. Intensity fluctuations}

We are interested in the spectrum $V(\omega)$ of the intensity fluctuations at the frequency $\omega=0$, as defined in Eq. (9). To obtain this spectrum at $\omega=0$ we start from Eqs. (33)-(35). We neglect the spontaneous emission in the laser mode and eliminate the rate equation for $N_{0}$ by using the assumption (35) that the total number of atoms is constant and is given by $N$. We linearize the remaining rate equations for $n, N_{1}$, and $N_{2}$ around the steady state by writing

$$
\begin{gathered}
n(t)=\bar{n}+\Delta n(t), \\
N_{1}(t)=\bar{N}_{1}+\Delta N_{1}(t), \\
N_{2}(t)=\bar{N}_{2}+\Delta N_{2}(t),
\end{gathered}
$$

and neglecting terms that are quadratic in the fluctuations. We take the Fourier transform of the linearized equations and solve for $\overline{\Delta n}(\omega)$, the Fourier transform of the photon number fluctuations $\Delta n(t)$. Because we are interested in the spectrum at zero frequency, we neglect the Fourier transforms of the time derivatives since they are proportional to $\omega$. We assume that $\bar{n} \gg 1$ and find

$$
\begin{gathered}
2 \kappa \overline{\Delta n}(\omega)=\left(1+\frac{1}{\beta \bar{n}}+\frac{\gamma_{0} A}{\gamma_{2} \beta \bar{n}}\right) \tilde{f}_{n}(\omega)+(A-1) \tilde{f}_{1}(\omega) \\
+A \widetilde{f}_{2}(\omega) \\
A=\frac{\gamma_{1}-\gamma_{2}}{\gamma_{1}+2 \gamma_{0}}
\end{gathered}
$$

where $\widetilde{f}_{i}(\omega)$ is the Fourier transform of $f_{i}(t)$. In Sec. II we introduced three uncorrelated noise sources in a laser, $f_{s t}$ for stimulated emission, $f_{a b s}$ for absorption, and $f_{v a c}$ for the fluctuations related to the emission of photons from the cavity. We distinguish three more noise sources, $f_{s p}^{1}$ and $f_{s p}^{2}$ for spontaneous emission in the 1-0 transition and 2-1 transition respectively, and $f_{\text {pump }}$ for the pump process. Their diffusion coefficients are given by [6]

$$
\begin{gathered}
D_{s p}^{1}=\gamma_{1} \bar{N}_{1}, \\
D_{s p}^{2}=\gamma_{2} \bar{N}_{2}, \\
D_{p u m p}=\gamma_{0} \bar{N}_{0} .
\end{gathered}
$$

The relation between the noise source $f_{n}$ and the uncorrelated noise sources has already been given in Eq. (6). For the noise sources $f_{i}$ with $i=0,1,2$ we have

$$
\begin{gathered}
f_{0}=f_{s p}^{1}-f_{\text {pump }}, \\
f_{1}=f_{s t}-f_{a b s}-f_{s p}^{1}+f_{s p}^{2},
\end{gathered}
$$

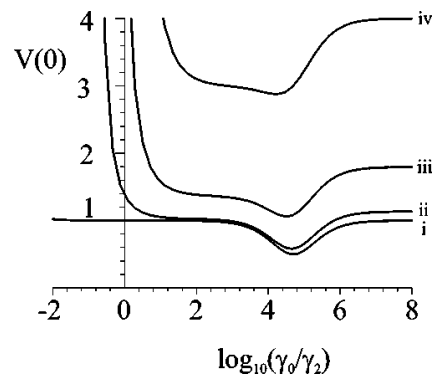

FIG. 5. Spectrum of intensity fluctuations at zero frequency $V(0)$ as a function of the pump rate $\gamma_{0}$ in units of $\gamma_{2}$. We have (i) $\varepsilon=0$, (ii) $\varepsilon=0.1$, (iii) $\varepsilon=0.3$, (iv) $\varepsilon=0.5$.

$$
f_{2}=-f_{s t}+f_{a b s}-f_{s p}^{2}+f_{\text {pump }} .
$$

From Eq. (7) it directly follows that

$$
\left\langle\tilde{f}_{i}(\omega) \tilde{f}_{j}\left(\omega^{\prime}\right)^{*}\right\rangle=D_{i j} \delta\left(\omega-\omega^{\prime}\right)
$$

By using Eq. (37) and the relation between the fluctuations in the photon number and the output intensity (10) we find for the spectrum defined in Eq. (9) at $\omega=0$ the following expression:

$$
\begin{aligned}
V(0)= & \frac{\gamma_{1}}{\gamma_{1}-\gamma_{2}} \frac{1+\beta \bar{n}}{\beta \bar{n}}\left(1+\frac{\gamma_{2}}{\gamma_{1}}-2 A+2 A^{2}\right) \\
& +\frac{1}{\beta \bar{n}}\left[1-A+A\left(\frac{\gamma_{0} A}{\gamma_{2}} \frac{1-\varepsilon}{\varepsilon}-\beta \bar{n}\right)\right] \\
& +\frac{2}{\beta^{2} \bar{n}^{2}}\left(\frac{\gamma_{1}+\gamma_{2} \beta \bar{n}}{\gamma_{1}-\gamma_{2}}\right)\left(1+\frac{\gamma_{0} A}{\gamma_{2}}\right)^{2}
\end{aligned}
$$

where $A$ is defined in Eq. (37).

With $\bar{n}$ given in Eq. (36) this quantity is displayed in Fig. 5 as a function of the pump rate for different values of $\varepsilon$. With our definition (9) the value $V(0)=1$ corresponds to shot noise. We see that for $\varepsilon \rightarrow 0$ and $\gamma_{0}$ fixed, which corresponds to the limit of the steady-state photon number $\bar{n}$ to infinity, the intensity fluctuations drop below shot noise if the pump rate becomes of the same order of magnitude as the decay rate $\gamma_{1}$ of level 1 . This result has been obtained before $[2,4]$. Hart and Kennedy [2] relate this reduction in noise to the accompanying depletion of the ground state, which reduces the pump noise. Ritsch and Zoller [4] focus on the regular recycling of the atoms in the pumping process, which leads to antibunching of the emission of the individual atoms and therefore to a reduction in the intensity noise. If $\varepsilon$ is increased we see that the divergence in noise that accompanies the threshold moves to higher pump rates. The noise increases for all pump rates and rises above shot noise, also in the range of pump rates where for $\varepsilon=0$ the intensity fluctuations are below shot noise.

Finally, applying the assumptions to the expression (36) for the steady-state number of photons in the laser mode, we find the same result as in Sec. III, Eq. (29). For the spectrum 
of intensity fluctuations at the frequency $\omega=0$ we find that the result from the semiclassical rate equations is

$$
V(0)=1+\frac{2 \varepsilon^{2}}{(1-\varepsilon)^{2}} \text {. }
$$

From the quantum-trajectory approach discussed in Sec. III we have Eq. (32),

$$
V(0)=1+\frac{2 \varepsilon}{(1-\varepsilon)^{2}} .
$$

We notice that the quantum-trajectory approach predicts more noise than the semiclassical description. This may be due to our ignoring other trajectories than the gain trajectory (with $k=0$ in Fig. 2). The atom number used in Eq. (26) only refers to the number of atoms in the gain trajectory. This underestimates the actual number of atoms, and therefore overestimates the noise.

\section{CONCLUSIONS}

We have analyzed a simple three-level model of a laser, where the number of active atoms $N$ is a limiting factor for laser action. A crucial role is played by the parameter $\varepsilon$, defined as the fraction of the total number of atoms in the gain medium that is needed for the laser to operate. The microscopic approach in Sec. III focuses on the pumping cycle of an individual atom using a quantum-trajectory picture. It is the dependence of the emission rate $f_{1}(\infty)$ or, equivalently, the cycle time $T_{0}$ on the number of photons in the laser mode that determines the statistics of the output of the laser. We find that far above the threshold, for pump rates $\gamma_{0}$ much smaller than the decay rate $\gamma_{1}$ of the lower lasing level, the output fluctuations increase if $\varepsilon$ is increased. We argue that the fraction of the total cycle time the atom spends in the ground state is $1-\varepsilon$. This explains the increase in intensity fluctuations for increasing value of $\varepsilon$. From the calculation of the Mandel $Q$ parameter we find that the emission of photons in the cavity mode by a single atom is antibunched and sub-Poissonian for $\varepsilon>0$. We conclude that an antibunched and sub-Poissonian emission of single atoms in the gain medium does not guarantee that the statistics of the output of the laser are below shot noise or sub-Poissonian.

From the macroscopic approach in Sec. IV we find that if $\varepsilon$ increases, the threshold of the laser moves to higher pump rates and the cycle time of the individual atoms increases, which makes the atoms less productive in creating photons. In the case that $\varepsilon=0$, the atoms on the ground state represent an infinite reservoir. In this limiting case, our results for the intensity fluctuations are in agreement with those of Hart and Kennedy [2] and Ritsch and Zoller [4]. For strong pumping, that is for values of the pump rate $\gamma_{0}$ of the same order of magnitude as the decay rate $\gamma_{1}$ of the lower level 1 of the lasing transition, the intensity fluctuations are below shot noise. However, if the parameter $\varepsilon$ is increased, the intensity fluctuations increase for all values of the pump rate $\gamma_{0}$. This is in agreement with the results from the microscopic approach. Hence, this scheme does not lead to an easy method to create sub-Poissonian laser radiation with a Poissonian pump of moderate strength.

\section{ACKNOWLEDGMENT}

This work is part of the research program of the 'Stichting voor Fundamenteel Onderzoek der Materie' (FOM).

\section{APPENDIX: MANDEL $Q$ PARAMETER}

Fluctuations in the number $m$ of photon emissions are commonly expressed in terms of the Mandel $Q$ parameter [15] defined by $Q+1=\Delta m^{2} /\langle m\rangle$, so that $Q=0$ for a Poisson process. We define $f_{2}\left(\tau_{2}, \tau_{1}\right) d \tau_{1} d \tau_{2}$ as the probability that there is an emission of a photon in both time intervals $\left(\tau_{2}, \tau_{2}+d \tau_{2}\right)$ and $\left(\tau_{1}, \tau_{1}+d \tau_{1}\right)$, regardless of how many photons have been emitted outside these time intervals. This function relates two events and can be interpreted as a correlation function. Since in our case the system is completely reset after each emission we can write

$$
f_{2}\left(\tau_{2}, \tau_{1}\right)=f_{1}\left(\tau_{2}-\tau_{1}\right) f_{1}\left(\tau_{1}\right) .
$$

We have [12]

$$
\frac{1}{2}\langle m(m-1)\rangle_{T}=\int_{0}^{T} d \tau_{2} \int_{0}^{\tau_{2}} d \tau_{1} f_{2}\left(\tau_{2}, \tau_{1}\right) .
$$

The Mandel $Q$ parameter in the steady state is then given by

$$
Q=\lim _{T \rightarrow \infty} \frac{2 \int_{0}^{T} d \tau_{2} \int_{0}^{\tau_{2}} d \tau_{1} f_{1}\left(\tau_{2}-\tau_{1}\right) f_{1}\left(\tau_{1}\right)-\int_{0}^{T} d \tau_{2} \int_{0}^{T} d \tau_{1} f_{1}\left(\tau_{2}\right) f_{1}\left(\tau_{1}\right)}{\int_{0}^{T} d \tau_{1} f_{1}\left(\tau_{1}\right)},
$$

where we used Eqs. (19), (A1) and (A2). From Eq. (19) we see that the integrals over $f_{1}$ are divergent for $T \rightarrow \infty$. Therefore we introduce the deviation $g_{1}\left(\tau_{1}\right)$ from the steady-state value as

$$
g_{1}\left(\tau_{1}\right)=f_{1}\left(\tau_{1}\right)-f_{1}(\infty) .
$$

The integrals over $g_{1}$ have an upper bound for $T \rightarrow \infty$. We can write the denominator in the expression (A3) for $Q$ as 


$$
\langle m\rangle_{T}=\int_{0}^{T} d \tau_{1} f_{1}\left(\tau_{1}\right)=T f_{1}(\infty)+\int_{0}^{T} d \tau_{1} g_{1}\left(\tau_{1}\right) .
$$

In the numerator the terms proportional to $T^{2}$ drop out and, if we take the limit $T \rightarrow \infty$, we find [16]

$$
Q=2 \hat{g}_{1}(0) \text {, }
$$

where $\hat{g}_{1}(s)$ is the Laplace transform of $g_{1}\left(\tau_{1}\right)$

$$
\hat{g}_{1}(s)=\int_{0}^{\infty} d \tau_{1} \exp \left(-s \tau_{1}\right) g_{1}\left(\tau_{1}\right)
$$

Using results from Sec. III we calculate $Q$. We apply the assumptions (27) and the expression (29) to find

$$
Q=-2 \varepsilon(1-\varepsilon)
$$

[1] M.O. Scully and M.S. Zubairy, Quantum Optics (Cambridge University Press, Cambridge, 1997), Chap. 12.

[2] D.L. Hart and T.A.B. Kennedy, Phys. Rev. A 44, 4572 (1991).

[3] G.A. Koganov and R. Shuker, Phys. Rev. A 63, 015802 (2000).

[4] H. Ritsch and P. Zoller, Phys. Rev. A 45, 1881 (1992).

[5] M.S. Kim and P.L. Knight, Phys. Rev. A 36, 5265 (1987).

[6] M. Lax, Phys. Rev. 145, 110 (1966).

[7] C.W. Gardiner and M.J. Collett, Phys. Rev. A 31, 3761 (1985).

[8] J. Dalibard, Y. Castin, and K. Molmer, Phys. Rev. Lett. 68, 580 (1992).

[9] R. Dum, P. Zoller, and H. Ritsch, Phys. Rev. A 45, 4879 (1992).
[10] H.J. Carmichael, An Open System Approach to Quantum Optics (Springer-Verlag, Berlin, 1993).

[11] C. Cohen-Tannoudji, B. Zambon, and E. Arimondo, J. Opt. Soc. Am. B 10, 2107 (1993).

[12] N.G. van Kampen, Stochastic Processes in Physics and Chemistry (North-Holland, Amsterdam, 1981), Chap. 2.

[13] G. Nienhuis, J. Stat. Phys. 53, 417 (1988).

[14] H. Risken, The Fokker-Planck Equation (Springer-Verlag, Berlin, 1996), Chap. 2.

[15] L. Mandel, Opt. Lett. 4, 205 (1979).

[16] H.F. Arnoldus and G. Nienhuis, Opt. Acta 30, 1573 (1983). 